\title{
Handwritten Chinese Character Recognition Using Displacement Extraction Based on Directional Features
}

\author{
Yoshiki Mizukami and Kazutoshi Koga \\ Faculty of Engineering, Yamaguchi University, \\ 2557 Tokiwadai, Ube, Yamaguchi 755, Japan
}

\begin{abstract}
This paper presents a recognition method for handwritten Chinese characters using displacement extraction based on directional features. In the proposed method, after extracting the features from an input image, the displacement is extracted by the minimization of an energy functional, which consists of the Euclidean distance and the smoothness of the extracted displacement. Experiments are performed on a set of handwritten Chinese characters (ETL-8B). The improvement of the recognition performance of the proposed method is achieved as compared with the method without the displacement extraction.
\end{abstract}

\section{Introduction}

The recognition of handwritten Chinese character has been studied by many researchers. However, it is still one of the difficult pattern recognition problems, because of the complex character shapes and a large number of categories as compared with the Roman alphabet.

In the design of an OCR (Optical Character Recognition) system, selection of a feature extraction method is important and many methods have already been proposed (e.g., Trier et al., 1996). The directional feature method, which utilizes directional factors of strokes composing a character, is considered a promising one and its efficiency has been clarified in previous works (Saito et al., 1982; Kimura and Shridhar, 1991). In general, by extracting features the dimensionality of the input data may be reduced. In addition to savings in computational costs, this has the advantage in the sense of avoiding the curse of dimensionality (Jain and Chandrasekaran, 1982).

In order to improve the recognition performance for deformed patterns, some approaches (Wakahara, 1994; Daffertshofer, 1994) have been attempted to extract the displacement presented in an input pattern. Yokoya (1994) has proposed a method for establishing dense matching of two views with large displacement by using the framework of regularization. Recently, Mizukami and Koga (1996) suggested a character recognition system, in which Yokoya's method is used to calculate the displacement of pixels on an input pattern, and the performance was demonstrated using a set of handwritten Roman characters in terms of the recognition rate. 


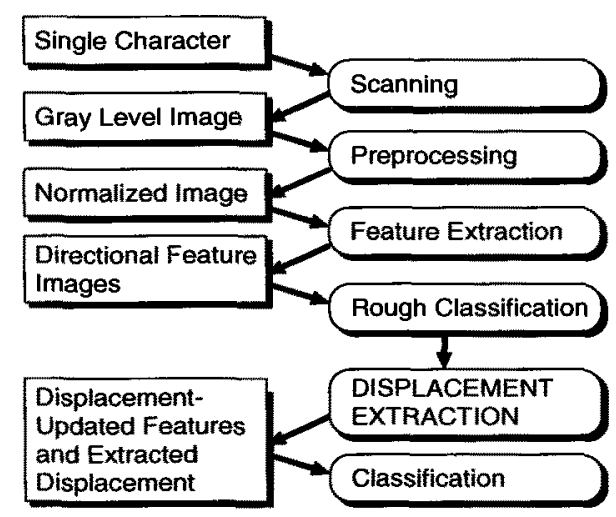

Fig. 1. Diagram of the proposed system.

In this paper a method using displacement extraction based on directional features is proposed for the purpose of improving the recognition performance for handwritten Chinese characters. In the method, after extracting features from an input image, the displacement is extracted based on them. In the classifcation module the resulting displacement is taken into account. To investigate the recognition performance, some experiments are performed on a data set of standard handwritten Chinese characters.

\section{Recognition System}

The proposed system consists of 6 modules: scanning, preprocessing, feature extraction, rough classification, displacement extraction and classification (Fig.1). The characteristic of the system is that the displacement extraction module is located between the modules of feature extraction and classification as compared with the traditional recognition system (e.g., Trier et al., 1996).

\subsection{Preprocessing}

First, the noise in the input image is removed by binarization. Next, the obtained binary image $f(x, y)$ (Fig.2(a)) is normalized so that the size of the rectangle circumscribing the pattern is $64 \times 64$ pixels. Consequently, the normalized image $f^{\prime}(x, y)$ is obtained (Fig.2(b)). This operation is described as follow:

$$
f^{\prime}(x, y)=f\left(\frac{w i d t h \times x}{64}+\delta x, \frac{h e i g h t \times y}{64}+\delta y\right)
$$

where width and height are that of the pattern, respectively. Then $\delta x$ and $\delta y$ are the horizontal and vertical distances between the left-top comers of the image and the rectangle, respectively. 


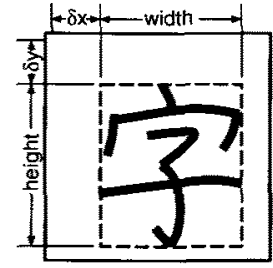

(a)

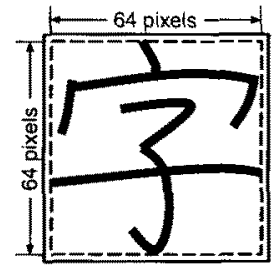

(b)

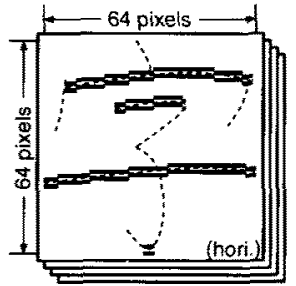

(c)

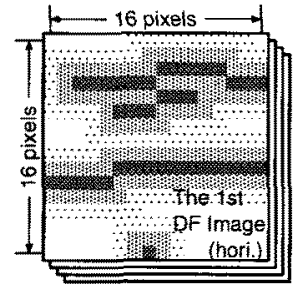

(d)

Fig. 2. Preprocessing and feature extraction. (a) Binary image: $f(x, y)$. (b) Normalized image: $f^{\prime}(x, y)$. (c) Contour images. (d) A set of directional feature images: $f_{k}(x, y)$, $k=1,2,3,4$.

Since it is inadequate for flat-shaped characters such as '-' to undergo the above described processing, if width $>3 \times$ height holds true, no normalization is applied.

\subsection{Feature Extraction}

In the feature extraction module, 4 feature images are generated from a normalized image $f^{\prime}(x, y)$. Each feature image contains different directional information of strokes, namely, horizontal $(\rightarrow)$, right-leaning $\left(\searrow_{\searrow}\right)$, vertical $(\downarrow)$ and left-leaning $(\swarrow)$ factors.

First, the direction of the contour is measured at each pixel on the contour of the pattern and the value 1 is put at the same coordinates on a contour image (64x64 pixels) corresponding to the obtained direction (Fig.2(c)). To cope with the noisy variations of the contour shape, the direction is calculated based on a line segment linking two coordinates, which are two-pixels on either side. If the obtained direction does not coincide with any of the four directions, then its value is distributed along the two near directions in an appropriate fashion.

Next, in order to reduce the number of features, sampling at $16 \times 16$ coordinates on each contour images is performed by averaging with a Gaussian filter $(\sigma=4)$. The obtained images (Fig.2(d)) are called as a set of directional feature images (DF images) and each of them is described as $f_{k}(x, y), k=1,2,3,4$.

\subsection{Rough Classification}

Regarding the recognition of Chinese character set that has many categories, the rough classification before the final one plays an important role in saving computational costs. In the rough classification module, 10 candidates are selected based on the Euclidean distances between an input set of DF images $f_{k}$ and each template set of DF images $g_{k}, k=1,2,3,4$. Note that we store one template for every category. 


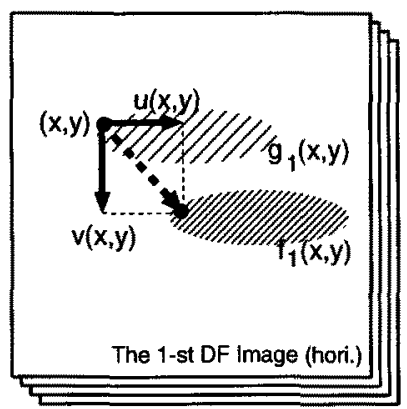

Fig. 3. Displacement functions $(u(x, y), v(x, y))$ between sets of DF images, $f_{k}(x, y)$ and $g_{k}(x, y)$.

\subsection{Displacement Extraction}

The displacement included in an input set of $f_{k}$ 's is extracted for each of the 10 candidate template sets of $g_{k}$ 's. It is necessary for the extracted displacement to satisfy the following two conditions:

1. The Euclidean distance between the two sets of DF images is reduced by the consideration of the extracted displacement.

2. The smoothness of the displacement is preserved.

Here two functions, $(u(x, y), v(x, y))$, are introduced (Fig.3). They represent the amount of the horizontal and vertical displacements at each coordinate on a set of DF images $g_{k}$, respectively. Then the displacement functions, which satisfy the above two conditions, should minimize an energy functional $E$ described as follows:

$$
E(u, v)=P(u, v)+\lambda S(u, v),
$$

where

$$
\begin{gathered}
P(u, v)=\iint \sum_{k}\left\{f_{k}(x+u(x, y), y+v(x, y))-g_{k}(x, y)\right\}^{2} d x d y \\
S(u, v)=\iint\left\{\left(\frac{\partial u(x, y)}{\partial x}\right)^{2}+\left(\frac{\partial u(x, y)}{\partial y}\right)^{2}+\left(\frac{\partial v(x, y)}{\partial x}\right)^{2}+\left(\frac{\partial v(x, y)}{\partial y}\right)^{2}\right\} d x d y .
\end{gathered}
$$

The penalty functional $P$ implies that the square of the Euclidean distance between the sets of DF images in consideration of the extracted displacement and the stabilization functional $S$ represents the measure of the departure from smoothness in the extracted displacement. Thus the minimization of these functionals corresponds to satisfying the above two conditions, respectively. The regularization parameter $\lambda$ controls the weight assigned to $S$ against $P$. 
The Euler-Lagrange equations of the energy functional $E$ are given as follows:

$$
\begin{aligned}
& \sum_{k}\left\{f_{k}(x+u(x, y), y+v(x, y))-g_{k}(x, y)\right\} \\
& \frac{\partial f_{k}(x+u(x, y), y+v(x, y))}{\partial x}-\lambda\left\{\frac{\partial^{2} u(x, y)}{\partial x^{2}}+\frac{\partial^{2} u(x, y)}{\partial y^{2}}\right\}=0, \\
& \sum_{k}\left\{f_{k}(x+u(x, y), y+v(x, y))-g_{k}(x, y)\right\} \\
& \frac{\partial f_{k}(x+u(x, y), y+v(x, y))}{\partial y}-\lambda\left\{\frac{\partial^{2} v(x, y)}{\partial x^{2}}+\frac{\partial^{2} v(x, y)}{\partial y^{2}}\right\}=0 .
\end{aligned}
$$

The Gauss-Seidel iteration equations at each pixel $(x, y)$ are obtained using finite difference approximations:

$$
\begin{array}{r}
u^{t+1}(x, y)=\bar{u}^{t}(x, y)-\frac{1}{4 \lambda} \sum_{k} \frac{\partial f_{k}\left(x+u^{t}(x, y), y+v^{t}(x, y)\right)}{\partial x} \\
\cdot\left\{f_{k}\left(x+u^{t}(x, y), y+v^{t}(x, y)\right)-g_{k}(x, y)\right\}, \\
v^{t+1}(x, y)=\bar{v}^{t}(x, y)-\frac{1}{4 \lambda} \sum_{k} \frac{\partial f_{k}\left(x+u^{t}(x, y), y+v^{t}(x, y)\right)}{\partial y} \\
\cdot\left\{f_{k}\left(x+u^{t}(x, y), y+v^{t}(x, y)\right)-g_{k}(x, y)\right\},
\end{array}
$$

where

$$
\begin{aligned}
& \bar{u}^{t}(x, y)=\frac{1}{4}\left\{u^{t}(x+1, y)+u^{t}(x-1, y)+u^{t}(x, y+1)+u^{t}(x, y-1)\right\}, \\
& \bar{v}^{t}(x, y)=\frac{1}{4}\left\{v^{t}(x+1, y)+v^{t}(x-1, y)+v^{t}(x, y+1)+v^{t}(x, y-1)\right\},
\end{aligned}
$$

and the superscript $t$ denotes the number of iterations.

\subsection{Classification}

Besides the functional $P$ that implies the Euclidean distance, the consideration of the functional $S$ is also advantageous for classification. The reason is that the displacement extracted for a set of DF images belonging to the same category would be more smooth as compared with a set of DF images belonging to different categories (Mizukami et al., 1996). In addition, the energy functional $E$, which consists of $P$ and $S$, is expected to be suitable for the classification. Thus each value of $E, P$ and $S$ is used as the distance between the patterns. 


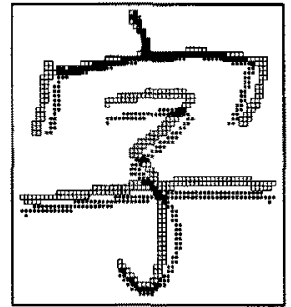

(a)

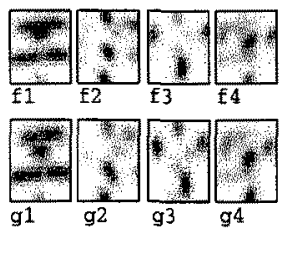

(b)

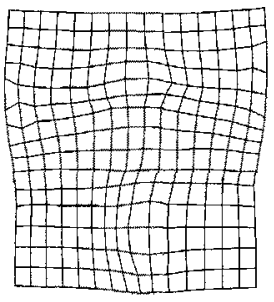

(c)

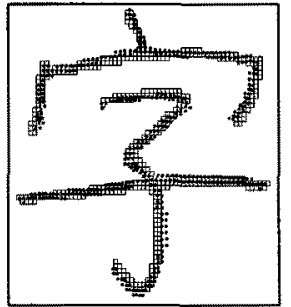

(d)

Fig. 4. Displacement extraction experiment. (a) Two Chinese characters: $f$ with $\square$ and $g$ with $\bullet$. (b) Sets of the DF images: $f_{k}$ 's and $g_{k}$ 's. (c) Extracted displacement: $u(x, y)$ and $v(x, y)$. (d) Displacement-updated pattern: $g(x, y)$.

\section{Experimental Results}

This section describes two experiments that were performed to investigate the proposed method. First, the experiment of displacement extraction using two Chinese characters is described in Section 3.1. Next, the recognition performance is clarified using ETL-8B in Section 3.2.

The regularization parameter $\lambda$ was set at 0.003 and all the initial values of $u$ and $v$ were set to 0 's. Equations (7) and (8) were iteratively used 20 times in extracting the displacement. Furthermore, for the improvement of the numerical stability, $\bar{u}$ and $\bar{v}$ of (9) and (10) were used as $u$ and $v$ in the second term of (7) and (8), respectively.

\subsection{Displacement Extraction Experiment}

In the proposed method the displacement is calculated indirectly from two sets of DF images, $f_{k}$ 's and $g_{k}$ 's, and not directly from two originals, $f$ and $g$, as described above. Thus the reliability of the displacement obtained in such a way should be confirmed. At the same time, the stability of the proposed method is discussed.

The displacement extraction experiment was performed using two Chinese characters (64x64 pixels) shown in Fig.4(a). The symbols, ' $\square$ ' and ' $\bullet$ ', represent the patterns $f$ and $g$, respectively. The two sets of DF images, $f_{k}$ 's and $g_{k}$ 's, are shown in Fig.4(b). Figure 4(c) illustrates the displacement extracted based on the DF sets with a graph net. Figure $4(d)$ shows the pattern $g$ deformed by the consideration of the obtained displacement using linear interpolation. In consideration of the promoted overlapping between originals, $f$ and $g$, the displacement obtained from the sets of DF images seems to be reliable.

Figure 5 shows changes in functionals $P, E$ and $S$ with respect to the number of iterations. After around 10 iterations, the variations of each functional became very small. The result shows a stable decrease of energy functional $E$. 


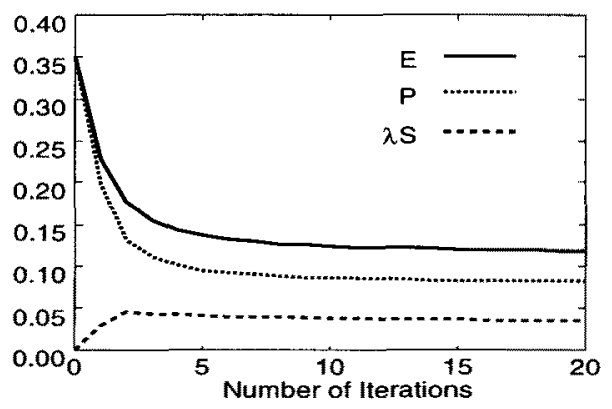

Fig. 5. Changes in $E, P$ and $S$.

\subsection{Recognition Experiment}

In this section the improvement of the recognition performance by the proposed method is investigated.

The ETL-8B has 881 categories of handwritten Chinese characters, and each category consists of 160 binary samples with a size of $64 \times 63$ pixels. Template sets of DF images $g_{k}$ were calculated using the odd-numbered samples of each category. Each template set of DF images was obtained by averaging the DF images belonging to the same category. The even-numbered samples were used as a test set $(70,480$ characters $)$.

Table 1 shows the recognition rates by using $E, P$ and $S$ as the distance. The result of the traditional method, in which no extraction of displacement was performed, is also shown for comparison. The cumulative recognition rate in the rough classification module was $98.65 \%$. The recognition rate with $P$ was $2.91 \%$ better than that of the traditional method, though the result with $S$ was $3.55 \%$ worse. Furthermore, an improvement of $3.96 \%$ was achieved by using the energy functional $E$, which is the weighted sum of $P$ and $S$. This indicates that the extraction of the displacement for a template belonging to the same category tends to reduce the Euclidean distance without any significant loss of smoothness.

It is confirmed that the displacement extraction based on sets of DF images is effective for improving the recognition performance.

Table 1. Recognition rates for ETL-8B.

\begin{tabular}{|l|l|l|l|l|}
\hline & Traditional & \multicolumn{4}{|c|}{ Displacement Extraction } \\
& Method & $\mathrm{P}$ & $\mathrm{S}$ & $\mathrm{E}$ \\
\hline Rate(\%) & 91.02 & $\mathbf{9 3 . 9 3}$ & 87.47 & 94.98 \\
\hline
\end{tabular}




\section{Conclusion}

This paper presented a recognition method for handwritten Chinese characters using displacement extraction based on directional features. In the proposed method, after extracting the features from an input image, the displacement is extracted by the minimization of an energy functional, which consists of the Euclidean distance and the smoothness of the extracted displacement.

Experiments were performed on a set of handwritten Chinese characters (ETL-8B). The improvement of the recognition performance of the proposed method is achieved as compared with the method without the displacement extraction.

\section{Future Work}

In this section some methods that would further improve the performance are described.

In the preprocessing module of the proposed system, the normalization is performed by a linear transformation, but some authors have demonstrated the effectiveness of nonlinear normalization methods (Yamada et al., 1990). The utilization of other local features should be investigated. Furthermore, the Mahalanobis distance, instead of the Euclidean distance, might be promising.

\section{References}

Trier, $\varnothing$. D., Jain, A. K. and Taxt, T.: Feature extraction methods for character recognition - A survey. Pattern Recognition, 29 (1996) 641-662

Saito, T., Yamada, H. and Yamamoto, K.: An analysis of handprinted Chinese characters by directional pattern matching approach. Trans. IECE (Japan), J65-D (1982) 550-557, in Japanese

Kimura, F. and Shridhar, M.: Handwritten numerical recognition based on multiple algorithms. Pattern Recognition, 24 (1991) pp. 969-983

Jain, A. K. and Chandrasekaran, B.: Dimensionality and sample size considerations in pattern recognition practice. Handbook of Statistics, Krishnaiah, P. R. and Kanal, L. N., eds, 2 (1982), North-Holland, Amsterdam

Wakahara, T.: Shape matching using LAT and its application to handwritten numeral recognition. IEEE Trans. Pattern Anal. Mach. Intell., 16 (1994) 618-629

Daffertshofer, A. and Haken, H.: A new approach to recognition of deformed patterns. Pattern Recognition, 27 (1994) 1697 1705

Mizukami, Y. and Koga, K.: A Handwritten character recognition system using hierarchical displacement extraction algorithm. in Proc. 13th Int. Conf. Pattern Recognition, 3 (1996) 160-164

Yokoya, N.: Dense matching of two views with large displacement. in Prc. 1th Int. Conf. Image Processing, 1 (1994) 213-217

Yamada, H., Yamamoto, K. and Saito, T.: A nonlinear normalization method for handprinted Kanji character recognition - line density equalization. Pattern Recognition, 23 (1990) 1023-1029 